\title{
Gal-1 regulates dendritic cells-induced Treg/Th17 balance though NF-кB/RelB-IL-27 pathway
}

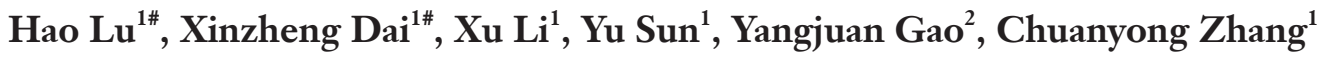 \\ ${ }^{1}$ Department of Liver Surgery, ${ }^{2}$ Department of Radiology, The First Affiliated Hospital of Nanjing Medical University, Nanjing 210000 , China \\ Contributions: (I) Conception and design: H Lu, C Zhang; (II) Administrative support: None; (III) Provision of study materials or patients: None; (IV) \\ Collection and assembly of data: H Lu, X Dai, X Li, Y Sun, Y Gao; (V) Data analysis and interpretation: H Lu, X Dai; (VI) Manuscript writing: All \\ authors; (VII) Final approval of manuscript: All authors. \\ \#These authors contributed equally to this work. \\ Correspondence to: Chuanyong Zhang. 300 Guangzhou Road, Nanjing 210000, China. Email: zcy2732@163.com.
}

\begin{abstract}
Background: This study aimed to investigate the mechanism of galectin (Gal)-1 of regulating Treg/Th17 in pathogenesis of acute rejection after liver transplantation in rat.

Methods: Mononuclear cells were induced to immature dendritic cells (imDCs), which were transfected

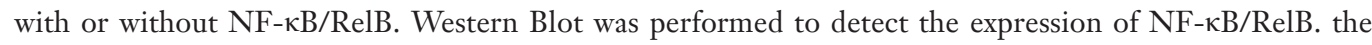
expression of CD11c, CD45RB, CD80 and MHC II were detected by flow cytometry. Enzyme-linked immunosorbent assay (ELISA) was employed to detect cytokines IL-27 and TGF- $\beta$. Lewis and dark agouti (DA) rats were generally anaesthetized by isoflurane inhalation to establish liver transplant models.

Results: We demonstrate that Gal-1 disturbs maturation of imDCs by downregulating NF- $\mathrm{KB} / \mathrm{RelB}$ expression, and Gal-1 negatively controls $\mathrm{CD}^{+}$proliferation though IL-27 pathway.

Conclusions: In aggregate, Gal-1 promotes Treg differentiation in $\mathrm{CD}^{+} \mathrm{T}$ cells though NF- $\kappa \mathrm{B} / \mathrm{RelB}-$ IL-27 pathway. These findings suggest a new therapeutic target to mediate Treg population.
\end{abstract}

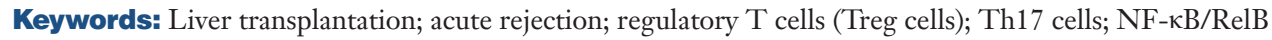

Submitted Jul 02, 2019. Accepted for publication Oct 10, 2019.

doi: $10.21037 /$ atm.2019.11.02

View this article at: http://dx.doi.org/10.21037/atm.2019.11.02

\section{Introduction}

Organ rejection which is activated by major histocompatibility complex (MHC) significantly limits the survival of transplant patients $(1,2)$. Regulatory $\mathrm{T}$ cells (Tregs) maintain immune homeostasis and induce immune tolerance (3). Studies have shown that therapeutic infusion of antigen-specific Tregs prolongs graft survival, and the number and function of Tregs in the recipient were inhibited when the rejection occurred $(4,5)$. Therefore, it is possible to prevent organ rejection by inducing immunology tolerance with antigen specific Tregs in recipients after organ transplantation.

Dendritic cells (DCs) play an indispensable role for Treg function in vivo (6). Researches show that the mature DCs, which are stimulated by special antigen, promote Treg differentiation and induce peripheral immune tolerance $(7,8)$. These DCs may be utilized for the treatment of autoimmune diseases and transplant rejection (9). One possible mechanism of DCs is that mature DC could be induced from immature DCs (imDCs) which uptake of apoptotic cell antigens, then the secretion of TGF- $\beta$ by mature DCs promotes the proliferation and activation of Treg, which results in immune tolerance $(10,11)$. Thus, understanding the influence mechanism of DCs-Treg becomes important for transplant rejection. RelB, a member of NF- $\mathrm{kB}$ family, plays a key role in the development of DCs and the DCs lack of RelB gene are unable to activate T cells $(12,13)$. However, the specific mechanisms of these DCs and $\mathrm{NF}-\kappa \mathrm{B} /$ RelB in immune balance remain controversial.

Galectin (Gal)-1 is an endogenous lectin and a member of the glycoside binding protein family. Gal-1 is involved in various biological processes including cell adhesion, apoptosis, proliferation and metastasis. Previous 
studies indicate that Gal-1 plays an important role in the proliferation and apoptosis of thymic and peripheral $\mathrm{T}$ cells (14-16). Further research shows that sera Gal-1 levels in organ transplant recipients were significantly higher than healthy controls (17). Thus, whether there is an association between Tregs and Gal-1 is worthy of attention. IL-27 is an indispensable immune cytokine which plays complex roles in $\mathrm{CD}^{+} \mathrm{T}$ cell subsets. IL-27 induces Th1 cell proliferation through STAT1 pathway while suppresses Th2/Th17 differentiation by targeting GATA3/STAT3 (18). Although IL-27 reduces the population of peripheral blood mononuclear cell (PBMC) in peripheral blood, it does benefit Treg proliferation (19). Previous study suggests that IL-27 blocks Th17 differentiation through STAT3-IL-10 pathway (20). Gal-1 pretreated DCs (DCs-Gal-1) are able to suppress DCs-induced lymphocyte proliferation and IFN- $\gamma$ production through IL-27 secretion (21). However, whether Gal-1 would have an impact on Treg/Th17 differentiation in $\mathrm{CD}^{+} \mathrm{T}$ cells remains unknown. Thus, we conducted this study to validate whether Gal-1 plays a role in DCs-induced Treg/Th17 balance through NF-kB/RelB-IL-27.

\section{Methods}

\section{Animal}

Lewis and dark agouti (DA) rats were purchased from The Beijing Vital River Laboratory, and housed in a specific pathogen-free facility in micro-isolator cages. Mice weighted $220 \pm 20 \mathrm{~g}$ were used. This study was carried out in accordance with the recommendations of the protocol (number NMU08-092) approved by the Institutional Animal Care and Use Committee of Nanjing Medical University.

\section{imDCs isolation and culture}

The Lewis rats were sacrificed by cervical dislocation. Mononuclear cells were obtained from femur and tibia by density gradient centrifugation and cultured in RPMI 1640 media containing $10 \%$ fetal bovine serum. Granulocytemacrophage colony-stimulating factor (GM-CSF) (Thermo Fisher, PRC2011) and IL-4 (Thermo Fisher, RRIL410) were added to the final concentration of $50 \mathrm{ng} / \mathrm{mL}$ and $25 \mathrm{ng} / \mathrm{mL}$, respectively.

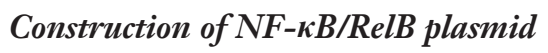

The corresponding primers and enzyme sites were designed based on the sequence of RelB in Genebank and amplified by PCR. The PCR products were purified and then connected to the LV5 vector. The positive clones were transformed and screened and then the plasmids were extracted. After sequencing, the results were verified for the subsequent experiments.

\section{Flow cytometry}

Rat-specific antibodies used for flow cytometry were purchased from BD Pharmingen, BioLegend and eBioscience. Acquisition was performed using a CATON (BD Bioscience) and data were analyzed using FlowJo software (TreeStar).

\section{Western blot and ELISA}

Cells were collected and lysed. The supernatant was centrifuged and quantified by BCA quantitative kit. The expression of $\mathrm{NF}-\kappa \mathrm{B} / \mathrm{RelB}$ was detected based on the Western blot protocol.

ELISA kits were purchased from $100 \mathrm{BIOTECH}$ and the samples were collected and assayed using ELISA protocol.

\section{Liver transplant models}

Cuff technique is used according to previous reports $(22,23)$. Briefly, both donor and recipient are generally anaesthetized by isoflurane inhalation. In donor part, large median and transverse incision was performed. Ligaments around liver were cut. A 24G, 4-mm-long stent was inserted into common bile duct. Proper liver artery was ligatured and not reconstructed. Graft was prepared on the back table and cold stored in University of Wisconsin solution (UW solution). Removal of native liver in recipient was similar to graft harvest, except the treatment of some blood vessels. Then, liver graft was implanted and the operation accomplished. A dose of $500 \mu \mathrm{g}$ recombinant Gal-1 was injected i.p. on post-operative day (POD) 0, 2, 4, 6 in Gal-1 treatment group.

\section{Statistical analyses}

RT-PCR data were analyzed using SDS v2.3 software. Survival data were analyzed using Prism 5 (Mantel-Cox). Other data were analyzed by analysis of variance (ANOVA) or Student $t$-test. Probability $(\mathrm{P})$ values less than or equal to 0.05 were considered statistically significant. 
A
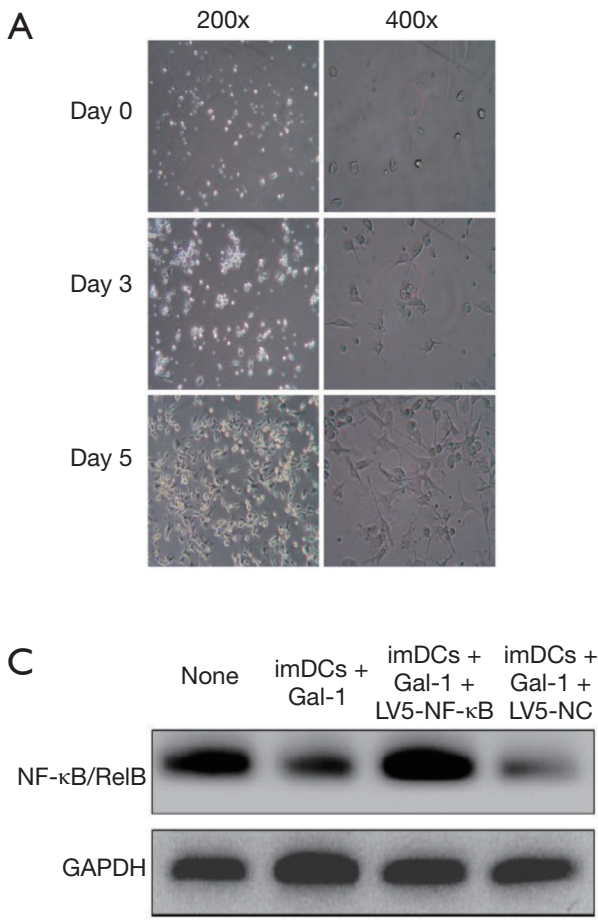

B

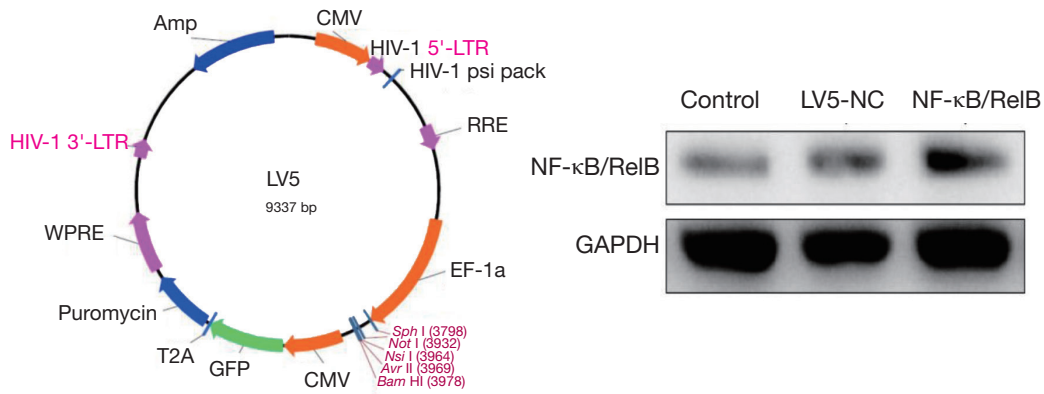

Figure 1 Gal-1 negatively regulates NF- $\kappa \mathrm{B} /$ RelB expression in imDCs $(\mathrm{n}=3)$. Mononuclear cells were obtained and then cultured in RPMI 1640 media containing $10 \%$ fetal bovine serum. GM-CSF $(50 \mathrm{ng} / \mathrm{mL})$ and IL-4 $(25 \mathrm{ng} / \mathrm{mL}$ ) were added to the final concentration. (A) The morphological changes of imDCs isolated from rats in 5 days; (B) lentivirus LV5 vector atlas was constructed as indicated (left). After $48 \mathrm{~h}$

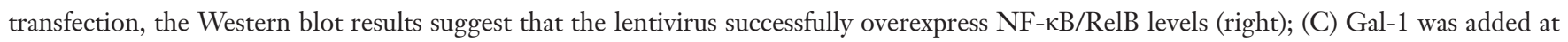
the beginning of transfection and cells were incubated in 4 different conditions (imDCs, imDCs + Gal-1, imDCs + Gal-1 + LV5-NF- $\kappa$ B/ RelB, imDCs + Gal-1 + LV5-NC) for 24 h more after transfection and then harvested for Western blot. NC, negative control; Gal, galectin; LTR, long terminal repeat; RRE, rev response element; imDCs, immature dendritic cells; GM-CSF, granulocyte-macrophage colonystimulating factor.

\section{Results}

\section{Gal-1 suppresses NF-кB/RelB expression in imDCs}

Mononuclear cells were obtained by density gradient centrifugation and cultured in RPMI 1640 media containing $10 \%$ fetal bovine serum. GM-CSF and IL-4 were added to the final concentration of 50 and $25 \mathrm{ng} / \mathrm{mL}$, respectively. Figure $1 \mathrm{~A}$ shows the morphological changes of imDCs isolated from rats in 5 days.

We constructed lentivirus LV5 vector atlas as indicated in Figure 1B. After $48 \mathrm{~h}$ transfection, as shown in Figure 1B, the Western blot results suggest that the lentivirus successfully overexpress NF- $\mathrm{kB} /$ RelB levels. To verify our hypothesis, Gal-1 was added at the beginning of transfection and cells were incubated in 4 different conditions (imDCs, imDCs + Gal-1, imDCs + Gal-1 + LV5-NF- $\kappa$ B/RelB, imDCs + Gal-1 + LV5-NC) for 24 h more after transfection and then harvested for protein assay. The result in Figure $1 C$ demonstrates that Gal-1 downregulates NF- $\mathrm{B} / \mathrm{RelB}$ expression in imDCs, while lentivirus reversed this effect.

\section{Gal-1 negatively controls the differentiation of imDCs into mature DCs by targeting $N F-\kappa B / R e l B$}

We next asked if Gal-1 would play a role in the differentiation of imDCs into mature DCs. CD11c, CD45RB, CD 80 and MHC II are the biomarkers of mature DCs. After transfection, we incubated these cells for $24 \mathrm{~h}$ more and then qualified protein expression by flow cytometry. Gal-1 treatment showed attenuated CD11c, CD45RB, CD80 and MHC II expression while the transfection of LV5-NF- $\mathrm{kB} /$ RelB rescued it, which indicates that imDCs maturation was suppressed by Gal-1 (Figure $2 A, B, C, D$ ). We further measured functional cytokines IL-27 and TGF- $\beta$. As indicated in 
A
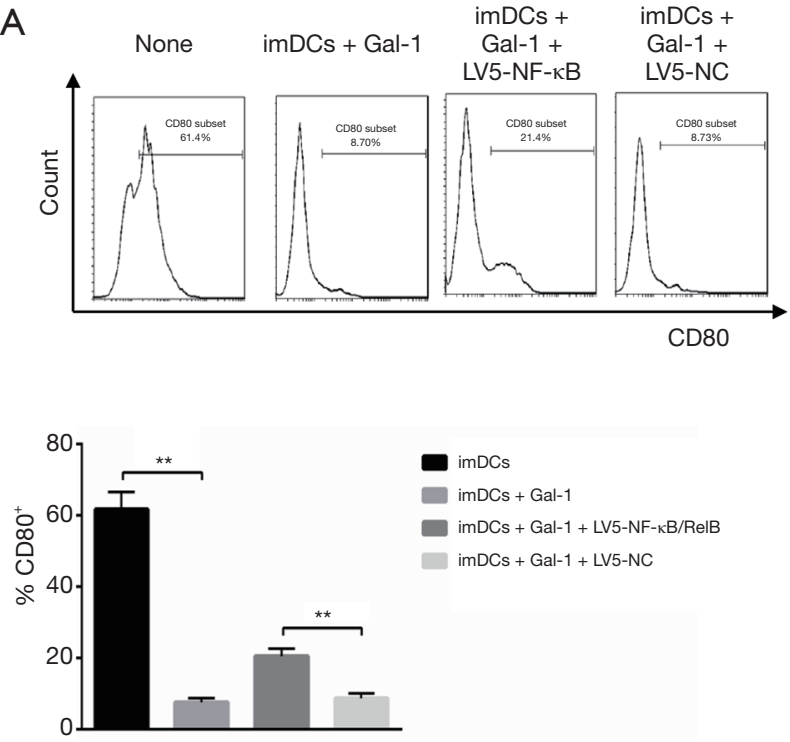

C
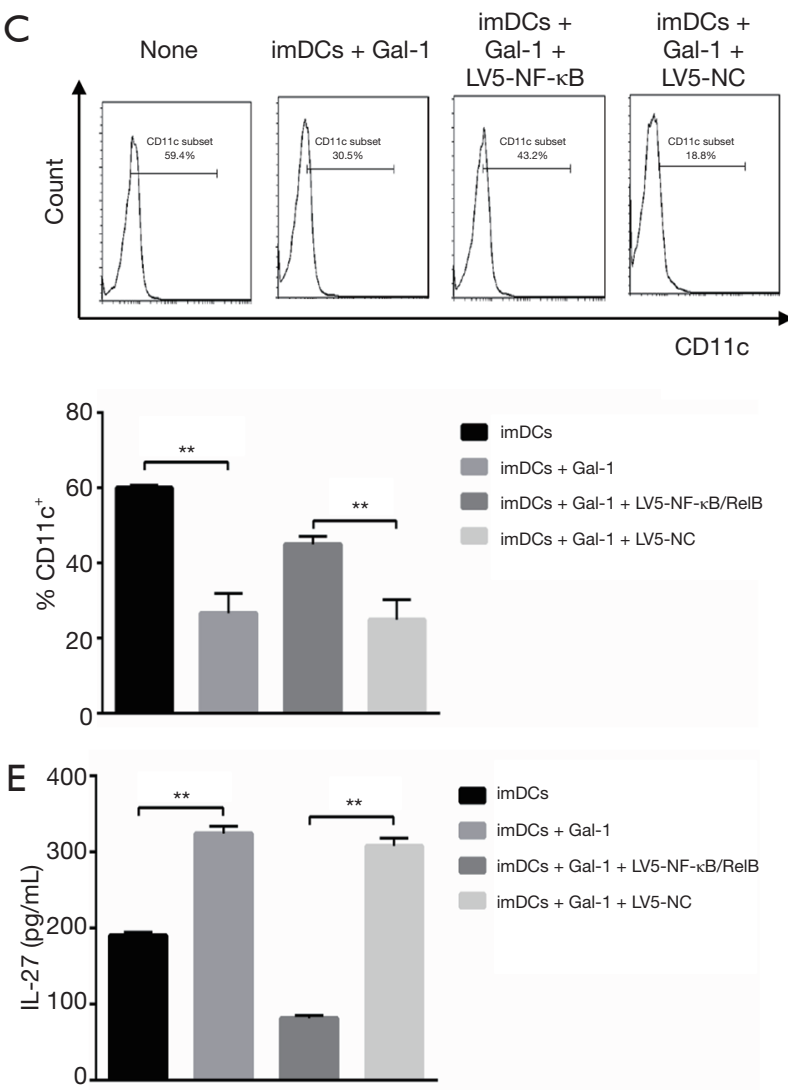

B
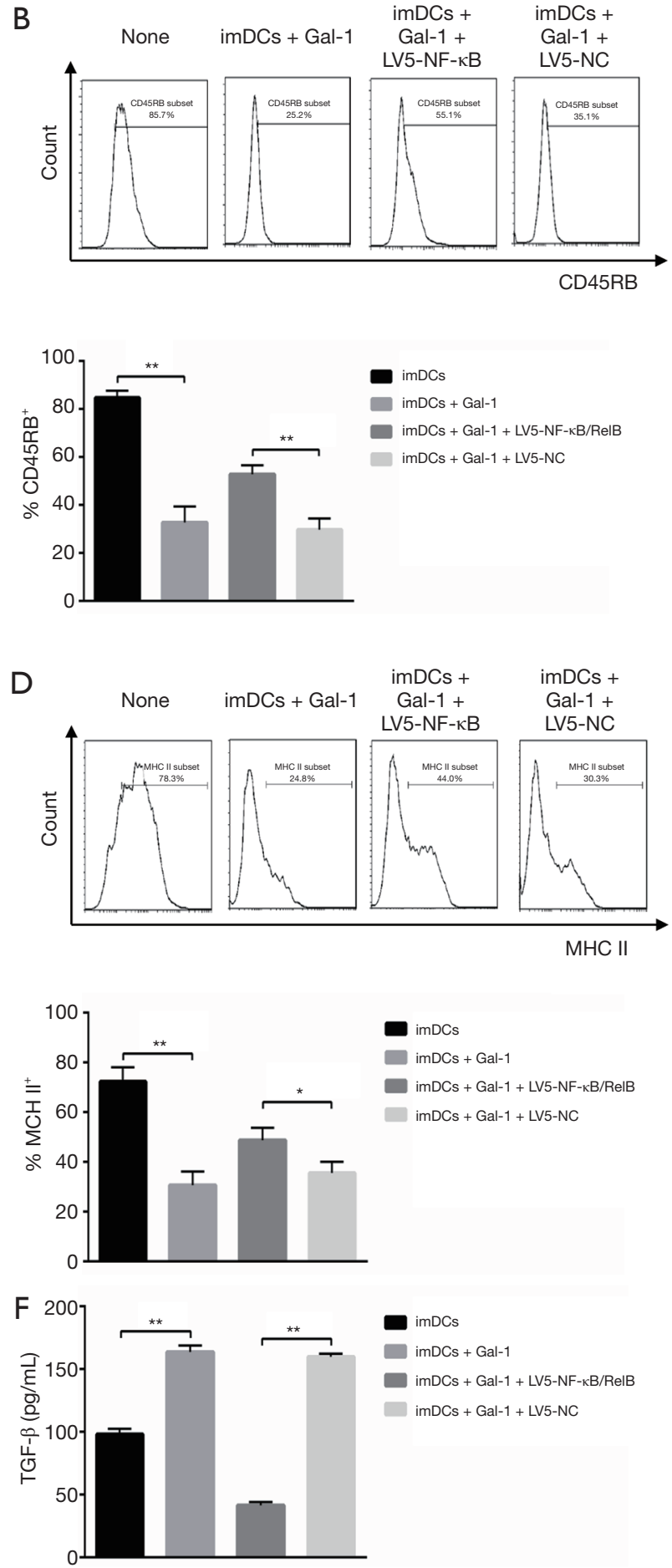

imDCs

imDCs + Gal-1

imDCs + Gal-1 + LV5-NF-kB/RelB

imDCs + Gal-1 + LV5-NC

MHC II

Figure 2 Gal-1 negatively controls the differentiation of imDCs into mature DCs by targeting NF- $\mathrm{kB} / \mathrm{RelB}(\mathrm{n}=3)$. After transfection, cells were incubated in 4 conditions as indicated for $24 \mathrm{~h}$ more and then assayed by flow cytometry. (A) CD80; (B) CD45RB; (C) CD11c; (D) MHC II expression and population were qualified. Cytokines such as (E) IL-27 and (F) TGF- $\beta$ were measured by ELISA. Values indicate mean \pm SEM of these experiments. *, $\mathrm{P}<0.05$; ${ }^{*}, \mathrm{P}<0.01$. imDCs, immature dendritic cells; NC, negative control; Gal, galectin; MHC, major histocompatibility complex; DC, dendritic cell; ELISA, enzyme-linked immunosorbent assay; SEM, standard error of mean. 

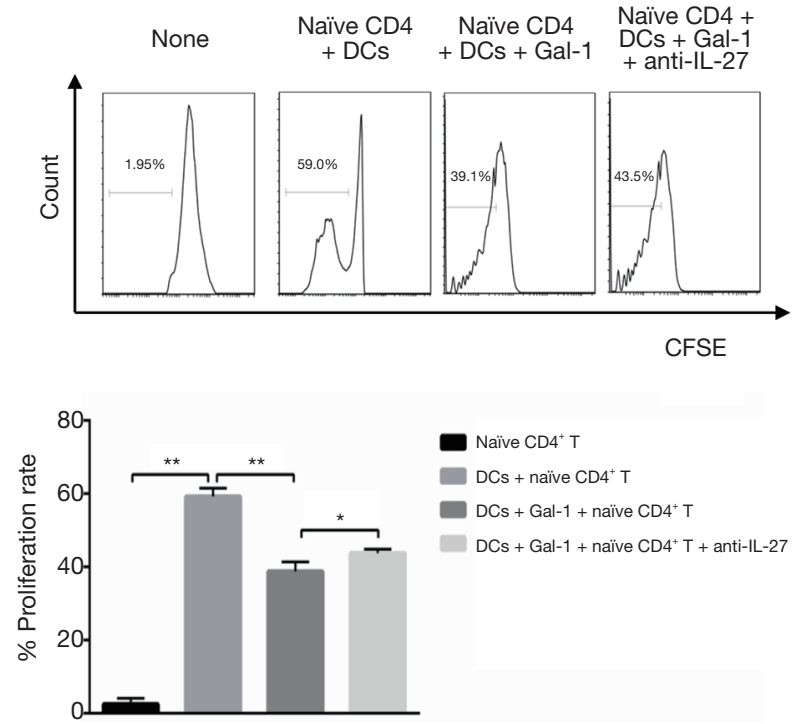

Figure 3 Gal-1 limits DCs-induced $\mathrm{CD}^{+} \mathrm{T}$ cells proliferation through IL-27 pathway $(n=3)$. Naïve $\mathrm{CD}^{+} \mathrm{T}$ cells from rats were sorted by flow cytometry (purity $\geq 94 \%$ ) and then the ability of proliferation was qualified by CFSE assay. $\mathrm{CD}^{+} \mathrm{T}$ cells co-cultured with or without DCs in 4 conditions, the CFSE fluorescence intensity and proliferation rate were qualified by flow assay. Values indicate mean \pm SEM of these experiments. ${ }^{*}, \mathrm{P}<0.05 ;{ }^{* *}, \mathrm{P}<0.01$. DCs, dendritic cells; Gal, galectin; CFSE, carboxyfluorescein succinimidyl ester; SEM, standard error of mean.

Figure 2E, $F$, up-regulated IL-27 and TGF- $\beta$ were detected in Gal-1 group while LV5-NF-кB/RelB demonstrated opposite effects. Taken together, Gal-1 treatment negatively regulates $\mathrm{NF}-\kappa \mathrm{B} / \mathrm{RelB}$, which is necessary for imDCs maturation.

\section{Gal-1 limits DCs-induced CD4 ${ }^{+}$T cells proliferation through IL-27 pathway}

Initially, we sorted $\mathrm{CD}^{+}{ }^{+} \mathrm{T}$ cells from rats by flow cytometry (purity $\geq 94 \%$ ). To ask whether Gal-1 would benefit cells expansion, the ability of proliferation was qualified by carboxyfluorescein succinimidyl ester (CFSE) assay. The result in Figure 3 showed that when naïve $\mathrm{CD}^{+}$ $\mathrm{T}$ cells co-cultured with DCs, the proliferative ability of naïve $\mathrm{CD}^{+} \mathrm{T}$ cells was remarkably improved. However, treatment of Gal-1 $(1 \mathrm{mg} / \mathrm{mL})$ weakened this effect but this effect was reversed by anti-IL-27. The results demonstrate that DCs promote $\mathrm{CD}^{+}{ }^{+} \mathrm{T}$ cells proliferation, and Gal-1 negatively controls $\mathrm{CD}^{+}{ }^{+}$proliferation through IL-27 pathway.

\section{Gal-1 controls the balance of DCs-induced Treg/Th17 through IL-27 pathway in $C D 4^{+} T$ cells}

After treatment in 4 conditions (none, DCs, DCs + Gal-1, DCs + Gal-1 + anti-IL-27) for 6 days in the presence of GM-CSF (50 ng/mL), Th17 (IL $\left.17^{+} \mathrm{CD} 4^{+}\right)$and Treg $\left(\mathrm{CD} 25^{+} \mathrm{FoxP}^{+}\right)$cell populations were qualified in $\mathrm{CD}^{+} \mathrm{T}$ cells by flow cytometry (Figure 4A,B), respectively. Th17 and Treg populations were increased when $\mathrm{CD}^{+} \mathrm{T}$ cells co-cultured with DCs. Treatment of Gal-1 attenuated Th17 differentiation but showed opposite effect for Treg cells, and addition of anti-IL-27 reversed this affection. Treg/Th17 ratios were further calculated in Figure $4 C$, which demonstrates that when co-cultured with DCs in the presence of Gal-1 $(1 \mathrm{mg} / \mathrm{mL}), \mathrm{CD}^{+} \mathrm{T}$ cells showed lower Th17 and higher Treg differentiation compared to control group. Addition of anti-IL-27 $(1 \mathrm{mg} / \mathrm{mL})$ eliminated the effect of Gal-1 and induced Th17 phenotype again. Cytokines such as IL-17, IL-10, TGF- $\beta$, IL-27 are necessary for Treg function. ELISA results in Figure 4D, E, F, G suggested that, in accordingly with cells differentiation, the secretion of IL-17, IL-10, TGF- $\beta$, IL-27 was obviously increased compared to control group. Treatment of Gal-1 $(1 \mathrm{mg} / \mathrm{mL})$ decreased IL-17 expression while addition of anti-IL-27 reversed this effect with attenuated IL-10, TGF- $\beta$, IL-27 secretion. To conclude, DCs induce naïve $\mathrm{CD}^{+} \mathrm{T}$ cells differentiation into Th17/Treg cells, and Gal-1 benefits Treg population through IL-27 signaling pathway.

\section{Gal-1 treatment significantly improves liver function and survival in allograft models}

Allograft causes high lethality compared to syngraft, thus we established rat liver transplant models to qualify the effect of Gal-1 in the treatment. Allograft or syngraft control liver transplant were performed as indicated with or without Gal-1 treatment. As shown in Figure 5A, allograft (allo) group showed reduced graft-versus-host disease (GVHD)induced lethality compared with syngraft group. And the treatment of Gal-1 in allograft significantly improved the survival. Consistent with survival, rat receiving allograft had highest levels of alanine transaminase (ALT) and lactate dehydrogenase (LDH) (Figure 5B,C), and Gal-1 treatment rescued liver function on day 3 . We further tested the expression of serum IL-17, IL-27 and IL-10 in vivo on day 3 . Although TGB- $\beta$ showed no difference, the results demonstrated similar results as in vitro with lower IL-17 
A

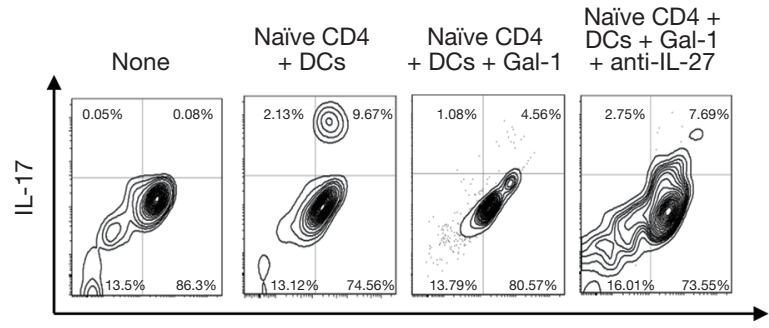

CD4
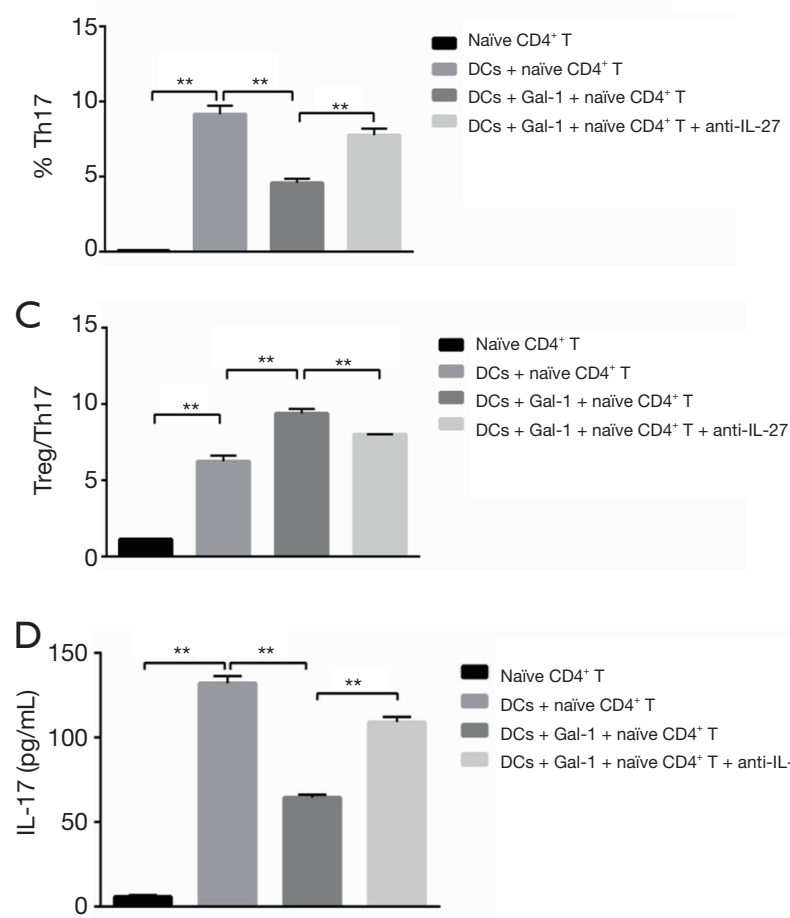

- Naïve $\mathrm{CD} 4^{+} \mathrm{T}$

DCs + naiive $C D 4^{+} T$

DCs + Gal-1 + naïve CD4 ${ }^{+} T$

DCs + Gal-1 + naïve CD4 $4^{+} \mathrm{T}+$ anti-IL-27

$\mathrm{F}$

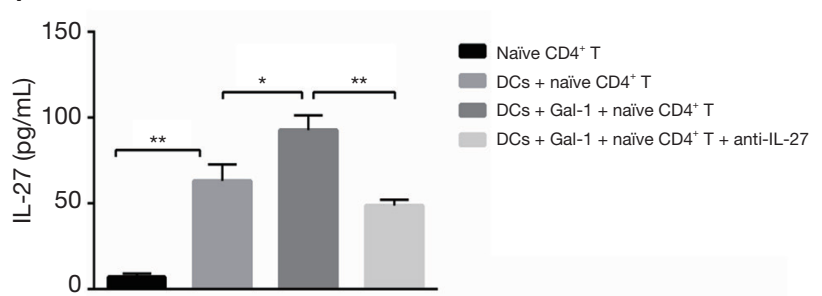

B
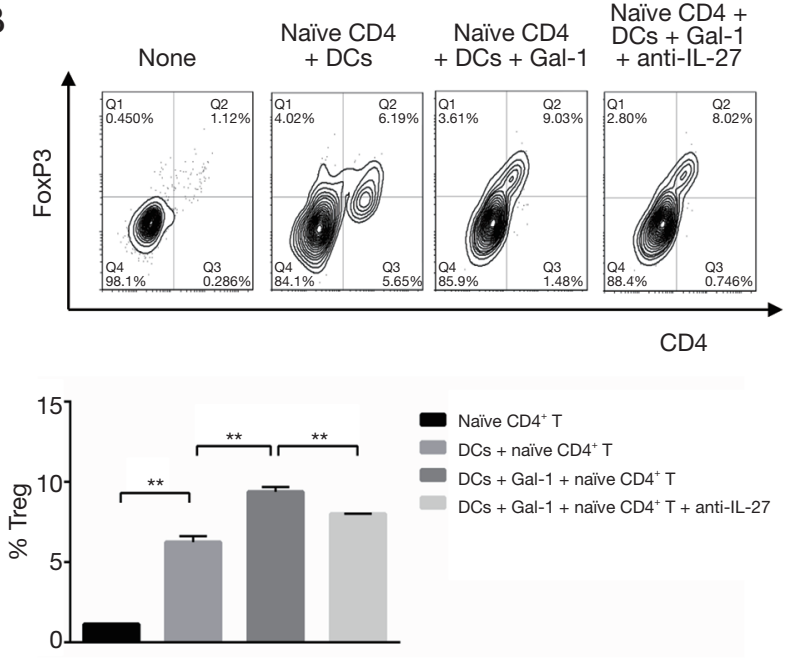

Naïve $C D 4^{+} T$

DCs + Gal-1 + naïve CD4 ${ }^{+} T$

DCs + Gal-1 + naïve CD4 ${ }^{+}$T + anti-IL-27
DCs + naiive $C D 4^{+} T$

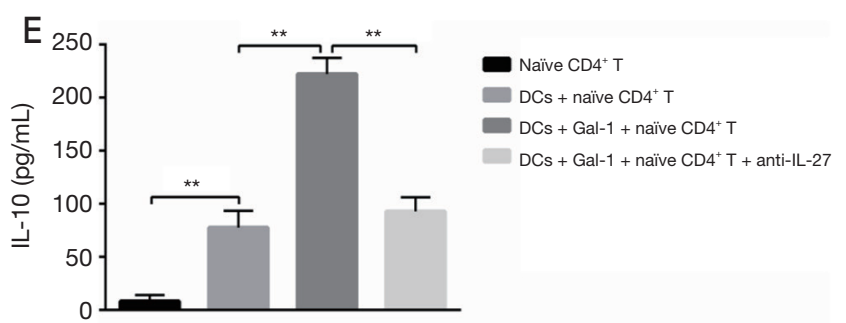

G

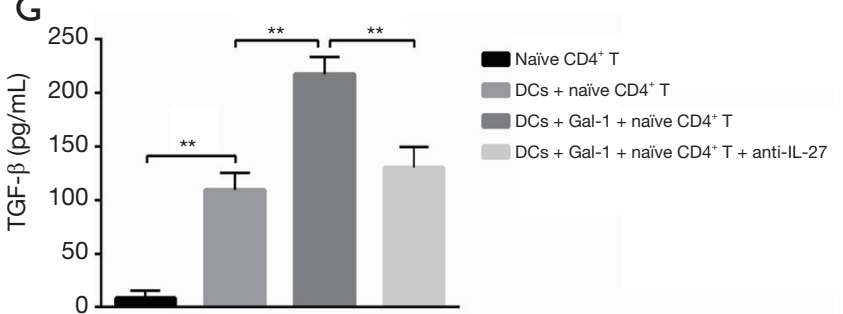

Figure 4 Gal-1 regulates DCs-induced Treg/Th17 balance through IL-27 pathway in CD4 ${ }^{+} \mathrm{T}$ cells ( $\left.\mathrm{n}=3\right)$. After treatment for 6 days in 4 conditions in the presence of GM-CSF $(50 \mathrm{ng} / \mathrm{mL})$, Th17 $\left(\mathrm{IL} 17^{+} \mathrm{CD}^{+}\right)(\mathrm{A})$ and $\mathrm{Treg}\left(\mathrm{CD} 25^{+} \mathrm{FoxP} 3^{+}\right)(\mathrm{B})$ cell populations were qualified in $\mathrm{CD}^{+} \mathrm{T}$ cells by flow cytometry. (C) Treg/Th17 ratios were further calculated. Cytokines such as IL-17 (D), IL-10 (E), IL-27 (F) and TGF- $\beta$ (G) were further measured by ELISA. Values indicate mean \pm SEM of these experiments. *, P<0.05; **, P<0.01. DCs, dendritic cells; Gal, galectin; GM-CSF, granulocyte-macrophage colony-stimulating factor; Treg cell, regulatory T cell; ELISA, enzyme-linked immunosorbent assay. 

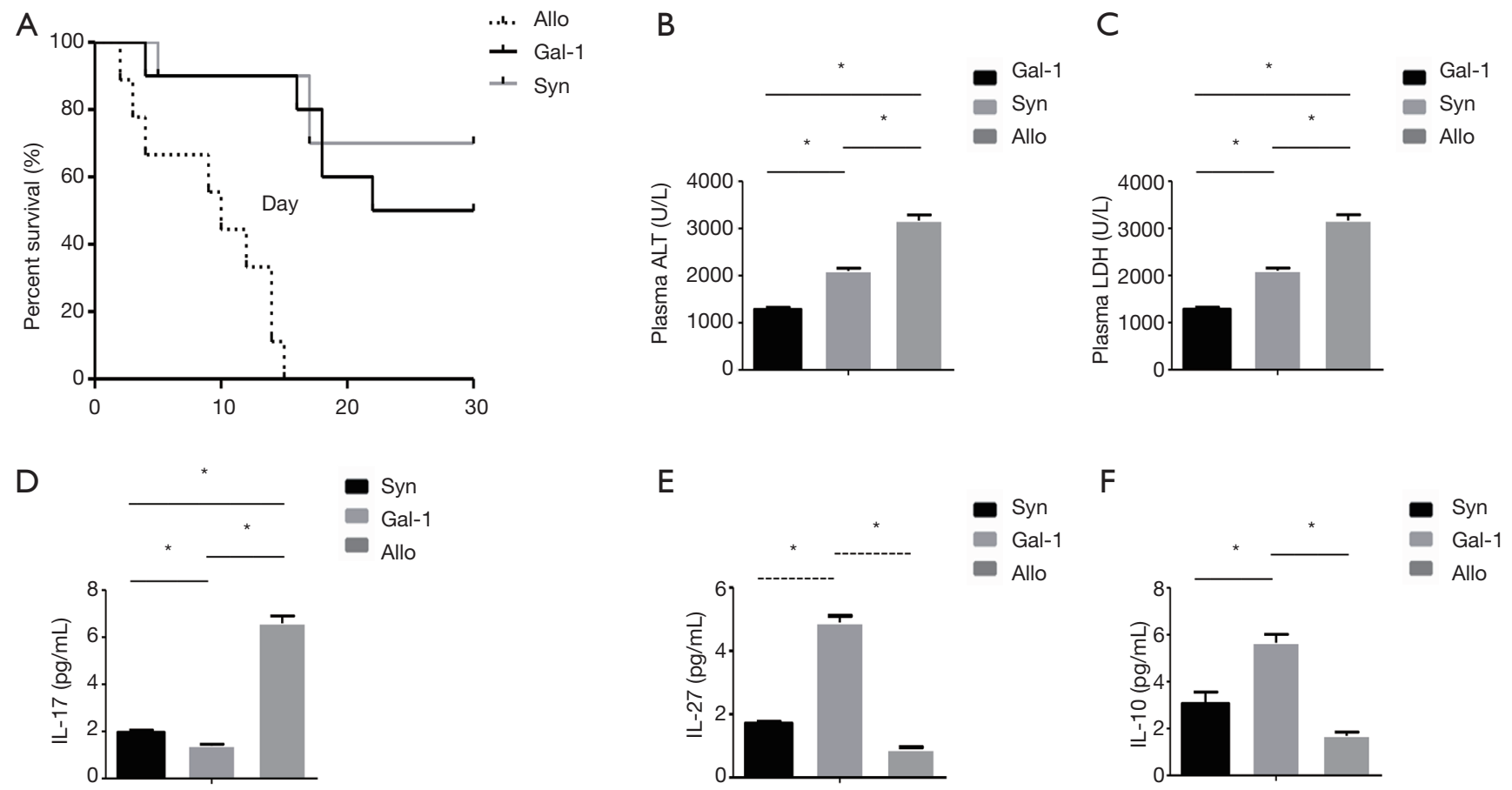

Figure 5 Gal-1 treatment significantly improves liver function and survival in allograft rat models (n=10). Liver transplant models were established to qualify the effect of Gal-1 in the treatment. Allograft or syngraft control liver transplant were performed as indicated with or without Gal-1 treatment. (A) Kaplan-Meier survival curves for rats receiving transplant surgery. Levels of ALT (B) and LDH (C) were measured for liver function on day 3 after surgery. IL-17 (D), IL-27 (E) and IL-10 (F) in serum were qualified by ELISA. Data shown are representative of 2 independent experiments. Values indicate mean \pm SEM of these experiments. * $\mathrm{P}<0.05$. Allo, allograft; Gal, galectin; Syn, syngraft; ALT, alanine transaminase; LDH, lactate dehydrogenase; ELISA, enzyme-linked immunosorbent assay; SEM, standard error of mean.

and higher IL-27, IL-10 expression (Figure 5D,E,F).

Thus, Gal-1 increases allograft liver transplant survival with better expression and can be exploited to improve the therapy for the prevention of human GVHD.

\section{Discussion}

Moderate Treg population is necessary for immune homeostasis. Previous studies have indicated that Treg could be induced into Th17 cells in inflammatory conditions and Treg/Th17 balance is associated with many immune diseases. In this study, we confirmed that: (I) Gal1 downregulates NF- $\kappa \mathrm{B} / \mathrm{RelB}$ expression in imDCs; (II) Gal-1 treatment negatively regulates NF- $\mathrm{KB} / \mathrm{RelB}$, which is necessary for imDCs maturation; (III) DCs promote $\mathrm{CD}^{+} \mathrm{T}$ cells proliferation, and Gal-1 negatively controls $\mathrm{CD}^{+}$proliferation through IL-27 pathway; (IV) DCs induce naïve $\mathrm{CD}^{+} \mathrm{T}$ cells differentiation into Th17/Treg cells, and Gal-1 benefits Treg population through IL-27 signaling pathway. Gal-1 promotes Treg differentiation in $\mathrm{CD}^{+} \mathrm{T}$ cells through NF-kB/RelB-IL-27 pathway. These findings suggest a new therapeutic target to mediate Treg population.

Gal-1 suppresses $\mathrm{T}$ cell activation by targeting $\mathrm{T}$ cell receptor (TCR): (I) restriction of TCR contact sites recombination; (II) blocking TCR signaling; (III) inactivation of T cells $(14,24)$. The role of IL-27 is more complicated because previous studies suggest that IL-27 can reduce Treg population by inhibiting its differentiation $(25,26)$. However, the recent study has shown that although IL-27 reduces T cell number in PBMC but it could promote Treg proliferation (19). Other studies also point out that the proliferation of Treg and inhibition of Th17 differentiation require IL-27 pathway (27-29). In our study, we show that Gal-1 negatively controls $\mathrm{CD} 4^{+}$proliferation but promotes Treg proliferation through IL-27 pathway. Furthermore, NF- $\kappa \mathrm{B} /$ RelB-IL-27 pathway in Treg is important for suppressive function and Gal-1 treatment 
might be a new method to improve Treg function for clinical trials.

The maturation of imDCs depends on proinflammatory stimulation and transcription factor NF- $\mathrm{KB}$, especially RelA (p65) and RelB (30-32). RelB knockout mice showed impaired antigen presenting cell (APC) function and cellular immunity $(12,33)$. We show that Gal-1 treatment downregulates NF- $\mathrm{\kappa B} /$ RelB expression, which might explain for the impaired imDCs maturation. Gal-1 pretreated DCs (DCs-Gal-1) also shows enhanced STAT3 phosphorylation level compared to control mature DCs. DCs-Gal-1-induced immune regulation could be attenuated by inhibition of STAT3, which results in inhibited proliferation of $\mathrm{CD} 4^{+}$ cells (34). Interestingly, DCs with silenced $N F-\kappa B / R e l B$ gene show the stronger ability to induce $\mathrm{CD} 4^{+} \mathrm{CD} 25^{+} \mathrm{T}$ cell $(21,35)$. These findings indicate that NF- $\kappa \mathrm{B} / \mathrm{RelB}$ is necessary for imDCs maturation but adverse for Treg cell induction, which might provide new insights into the area of Treg expansion. Thus, Gal-1 inhibits NF-кB/RelB activation in DCs, and DCs-secreted IL-27 induces $\mathrm{CD}^{+}$ $\mathrm{T}$ cells to Treg cells and changes the balance of Treg/Th17 after transplantation.

In this study, we have shown that Gal-1 treatment in vitro is an easy and efficient way of increasing the population of Treg cells, which may be useful in clinical applications. In summary, we demonstrate that Gal-1 regulates NF$\kappa B /$ RelB-IL-27 pathway, which plays a vital role in tTreg expansion, and focusing on this pathway might become a new starting point in Treg clinical trials.

\section{Acknowledgments}

Funding: This work was supported in part by the National Nature Science Foundation of China (No. 81571559) and the Natural Science Foundation of Jiangsu Province (No. BK20151021) in China.

\section{Footnote}

Conflicts of Interest: The authors have no conflicts of interest to declare.

Ethical Statement: The authors are accountable for all aspects of the work in ensuring that questions related to the accuracy or integrity of any part of the work are appropriately investigated and resolved. This study was carried out in accordance with the recommendations of the protocol (number NMU08-092) approved by the
Institutional Animal Care and Use Committee of Nanjing Medical University.

\section{References}

1. Huang HF, Zeng Z, Chen MQ. Roles of Kupffer cells in liver transplantation. Hepatogastroenterology 2012;59:1251-7.

2. Burwitz BJ, Wu HL, Abdulhaqq S, et al. Allogeneic stem cell transplantation in fully MHC-matched Mauritian cynomolgus macaques recapitulates diverse human clinical outcomes. Nat Commun 2017;8:1418.

3. Kraj P, Ignatowicz L. The mechanisms shaping the repertoire of CD4+ Foxp3+ Treg cells. Immunology 2018;153:290-6.

4. Lee K, Nguyen V, Lee KM, et al. Attenuation of donorreactive $\mathrm{T}$ cells allows effective control of allograft rejection using regulatory T cell therapy. Am J Transplant 2014;14:27-38.

5. Zhou Y, Yang X, Zhang H, et al. The roles of $\mathrm{T}$ helper type 17/regulatory $T$ cells in acute rejection after liver transplantation in rats. Transplantation 2015;99:1126-31.

6. Guermonprez P, Valladeau J, Zitvogel L, et al. Antigen presentation and $\mathrm{T}$ cell stimulation by dendritic cells. Annu Rev Immunol 2002;20:621-67.

7. Morelli AE, Thomson AW. Tolerogenic dendritic cells and the quest for transplant tolerance. Nat Rev Immunol 2007;7:610-21.

8. da Costa TB, Sardinha LR, Larocca R, et al. Allogeneic apoptotic thymocyte-stimulated dendritic cells expand functional regulatory $\mathrm{T}$ cells. Immunology 2011;133:123-32.

9. Zhou F, Lauretti E, di Meco A, et al. Intravenous transfer of apoptotic cell-treated dendritic cells leads to immune tolerance by blocking Th17 cell activity. Immunobiology 2013;218:1069-76.

10. Huang H, Dawicki W, Zhang X, et al. Tolerogenic dendritic cells induce CD4+CD25hiFoxp3+ regulatory $\mathrm{T}$ cell differentiation from CD4+CD25-/loFoxp3- effector T cells. J Immunol 2010;185:5003-10.

11. Gleisner MA, Rosemblatt M, Fierro JA, et al. Delivery of alloantigens via apoptotic cells generates dendritic cells with an immature tolerogenic phenotype. Transplant Proc 2011;43:2325-33.

12. Wu L, D'Amico A, Winkel KD, et al. RelB is essential for the development of myeloid-related CD8alpha- dendritic cells but not of lymphoid-related CD8alpha+ dendritic cells. Immunity 1998;9:839-47. 
13. Qiu T, Zhu HC, Liu XH, et al. Lentiviral-mediated shRNA against RelB induces the generation of tolerogenic dendritic cells. Int Immunopharmacol 2012;12:501-9.

14. Elola MT, Chiesa ME, Alberti AF, et al. Galectin-1 receptors in different cell types. J Biomed Sci 2005;12:13-29.

15. Camby I, Le Mercier M, Lefranc F, et al. Galectin-1: a small protein with major functions. Glycobiology 2006;16:137R-157R.

16. St-Pierre C, Ouellet $M$, Tremblay MJ, et al. Galectin-1 and HIV-1 Infection. Methods Enzymol 2010;480:267-94.

17. García MJ, Jurado F, San Segundo D, et al. Galectin-1 in stable liver transplant recipients. Transplant Proc 2015;47:93-6.

18. Yoshimoto T, Yasuda K, Mizuguchi J, et al. IL-27 suppresses Th2 cell development and Th2 cytokines production from polarized Th2 cells: a novel therapeutic way for Th2-mediated allergic inflammation. J Immunol 2007;179:4415-23.

19. Hall AO, Beiting DP, Tato C, et al. The cytokines interleukin 27 and interferon-gamma promote distinct Treg cell populations required to limit infection-induced pathology. Immunity 2012;37:511-23.

20. Stumhofer JS, Laurence A, Wilson EH, et al. Interleukin 27 negatively regulates the development of interleukin 17-producing $\mathrm{T}$ helper cells during chronic inflammation of the central nervous system. Nat Immunol 2006;7:937-45.

21. Ilarregui JM, Croci DO, Bianco GA, et al. Tolerogenic signals delivered by dendritic cells to $T$ cells through a galectin-1-driven immunoregulatory circuit involving interleukin 27 and interleukin 10. Nat Immunol 2009;10:981-91.

22. Winearls CG, Fabre JW, Millard PR, et al. A quantitative comparison of whole antibody and $\mathrm{F}\left(\mathrm{ab}^{\prime}\right) 2$ in kidney allograft enhancement. Transplantation 1979;28:36-9.

23. Oldani G, Lacotte S, Morel P, et al. Orthotopic liver transplantation in rats. J Vis Exp 2012. doi: 10.3791/4143.

24. Chung CD, Patel VP, Moran M, et al. Galectin-1 induces partial TCR zeta-chain phosphorylation and antagonizes processive TCR signal transduction. J Immunol

Cite this article as: Lu H, Dai X, Li X, Sun Y, Gao Y, Zhang C. Gal-1 regulates dendritic cells-induced Treg/Th17 balance though NF-кB/RelB-IL-27 pathway. Ann Transl Med 2019;7(22):628. doi: 10.21037/atm.2019.11.02
2000;165:3722-9.

25. Huber M, Steinwald V, Guralnik A, et al. IL-27 inhibits the development of regulatory $\mathrm{T}$ cells via STAT3. Int Immunol 2008;20:223-34.

26. Cox JH, Kljavin NM, Ramamoorthi N, et al. IL-27 promotes $\mathrm{T}$ cell-dependent colitis through multiple mechanisms. J Exp Med 2011;208:115-23.

27. Xu J, Yang Y, Qiu G, et al. c-Maf regulates IL10 expression during Th17 polarization. J Immunol 2009;182:6226-36.

28. Iwasaki Y, Fujio K, Okamura T, et al. Egr-2 transcription factor is required for Blimp-1-mediated IL-10 production in IL-27-stimulated CD4+ T cells. Eur J Immunol 2013;43:1063-73.

29. Kim G, Shinnakasu R, Saris CJ, et al. A novel role for IL27 in mediating the survival of activated mouse CD4 T lymphocytes. J Immunol 2013;190:1510-8.

30. Rescigno M, Martino M, Sutherland CL, et al. Dendritic cell survival and maturation are regulated by different signaling pathways. J Exp Med 1998;188:2175-80.

31. Ardeshna KM, Pizzey AR, Devereux S, et al. The PI3 kinase, p38 SAP kinase, and NF-kappaB signal transduction pathways are involved in the survival and maturation of lipopolysaccharide-stimulated human monocyte-derived dendritic cells. Blood 2000;96:1039-46.

32. Gerondakis S, Banerjee A, Grigoriadis G, et al. NFkappaB subunit specificity in hemopoiesis. Immunol Rev 2012;246:272-85

33. Luo L, Sun Z, Fang Q, et al. Effects of tolerogenic dendritic cells generated by siRNA-mediated RelB silencing on immune defense and surveillance functions of T cells. Cell Immunol 2013;282:28-37.

34. Rabinovich GA, Gabrilovich D, Sotomayor EM. Immunosuppressive strategies that are mediated by tumor cells. Annu Rev Immunol 2007;25:267-96.

35. Misra N, Bayry J, Lacroix-Desmazes S, et al. Cutting edge: human CD4+CD25+ T cells restrain the maturation and antigen-presenting function of dendritic cells. J Immunol 2004;172:4676-80 